\section{Speciation in the 21st century}

\author{
Speciation \\ JA Coyne and H Allen Orr \\ Sinauer Associates Inc., Massachusetts, USA; 2004. 545 pp. \\ $£ 34.99$, paperback. ISBN 0878930892. \\ Heredity (2005) 95, 105-109. doi:10.1038/sj.hdy.6800686
}

Reviewed by J Mallet

So effusive was Axel Meyer's appreciation of 'Speciation' in 'Nature' that the review seemed almost obsequious. In 'Science', Benjamin Blackman and Loren Rieseberg were more circumspect, but came to a similar conclusion: that this book is a landmark publication representing the state of the art in speciation research today. The critical acclaim for Coyne and Orr's 'Speciation' is, in my view, fully warranted. Consisting of 545 closely packed pages, it contains an up-to-date and very complete summary of empirical and theoretical studies about speciation, and reviews publications dating from Darwin and before, from the 1930-1950 'modern synthesis', and up to the present day. A not inconsiderable number of unpublished manuscripts are also referenced. 'Speciation' will be useful for years to come. All evolutionary biologists should buy it.

Unlike my eminent colleagues writing in posher journals, I will now concentrate on some criticisms. Instead of taking the opportunity to re-think the whole field, it seems to me that Coyne and Orr have dusted off ideas arising in the 1930s and 1940s, while Hitler, Stalin, Mussolini and Franco ruled much of Europe. It is probably a good idea to test such views from time to time. Therefore, although I again emphasize that I regard 'Speciation' as one of the most balanced and thoughtful books on the topic to date, and although most of us agree with most of their conclusions, I will use the remainder of the review to develop a critique of one or two problems left outstanding.

Some views that characterize the Dobzhansky-Mayr period of the 1930s and 1940s are (1) that Darwin wrote a nice book on natural selection, but largely ignored speciation, (2) that species are 'real', and qualitatively different from lower or higher taxa, (3) that the 'biological species concept' is the only useful way of defining species, especially when studying speciation, and (4) that speciation will occur much more easily in geographic isolation (allopatry) than between populations in contact, and that allopatric speciation can be used as a kind of 'null hypothesis', to be accepted unless disproved by watertight evidence of alternatives. Coyne and Orr support all four of these views.

As I have argued elsewhere, these and similar mid20th century evolutionary ideas are now coming under much more attack than they were 30 years ago, and indeed the extensive discussions of points 2-4 in this book show that, far from ignoring the recent literature, Coyne and Orr have been strongly influenced by new data and theory in these topics. However, the authors do develop detailed arguments against the newer work in an attempt to shore up the Dobzhansky-Mayr tradition.

I discuss each of these Coyne and Orr views in turn.

\section{(1) Darwin's views on species and speciation}

Perhaps it doesn't matter for the progress of science what Darwin actually thought, but after about 100 years of Darwin-bashing, can we maybe give the man a break? Is it really true that 'his magnum opus remains largely silent on the 'mystery of mysteries', and the little it does say about this mystery is seen by most modern evolutionists as muddled or wrong' (Coyne and Orr, p 9)? It was after all Darwin's own astonishing clarity of vision and resistance to educational brainwashing that more or less initiated the study of speciation.

Mayr (1942, p 147) used similar arguments to justify his own vision of speciation: 'Darwin's book was misnamed, because it is a book on evolutionary changes in general and the factors that control them (selection and so forth), but not a treatise on the origin of species'. However, this, it seems to me, is pure hindsight. Supposing you adopt a non-Darwinian view of species, that species are 'real', and utterly distinct from higher or lower taxa, and that speciation is mainly caused by geographical isolation rather than natural selection, then, obviously, Darwin's ideas - that species are just rather strongly differentiated varieties with few intermediates, which are produced by divergent natural selection would not seem to be about speciation any more.

In any case, although the relative weight of arguments may have changed since the time of Darwin, he simply didn't say that reproductive isolation had nothing to do with species, nor did he argue that species were unreal in the sense that you couldn't tell them apart locally (as a naturalist he knew better), and nor did he argue that speciation had nothing to do with geography or geographic isolation. Quite the reverse: 'The fertility of varieties ... is, on my theory, of equal importance with the sterility of species; for it seems to make a broad and clear distinction between varieties and species' (Darwin, 1859, p 246). 'Finally, then, varieties have the same general characters as species, for they cannot be distinguished from species, - except, firstly, by the discovery of intermediate linking forms ...' (Darwin, 1859, p 58). '[Geographic] Isolation, also, is an important element in the process of natural selection.... Although I do not doubt that [geographic] isolation is of considerable importance in the production of new species, on the whole I am inclined to believe that largeness of area is of more importance' (Darwin, 1859, pp 104-106).

Darwin thus disagreed quantitatively, rather than qualitatively, with the later Mayr-Dobzhansky analyses of species and speciation. On species concepts, he felt that reproductive isolation was important, but was not an 'essential' difference in distinguishing species (Darwin, 1859, pp 52, 246-278). All Darwin is really saying here is that there is no theoretical barrier for 'varieties' to evolve into 'species', since they are the same kinds of things. At root, all evolutionary biologists agree with this position, surely! 
It is certainly true that Darwin advocated the idea that speciation could take place in the presence of gene flow, on the grounds that large continents were usually more speciose than adjacent islands. Darwin also used the theoretical arguments that more biotic interactions would be present within continents, and that more opportunity existed for variations (mutations) to arise in larger populations. I don't think that this necessarily advocates 'sympatric' speciation in today's terms. A related issue is Darwin's 'principle of divergence', in which he argues that speciation is driven, if you like, by survival of recently diverged populations in new niches and the extinction of less-well-adapted intermediates. It is clear that he doesn't have a detailed mechanism to explain how this occurs, but this is hardly surprising given that we now know he had an erroneous view of heredity.

In conclusion, one may criticize Darwin for not having discovered genetics, and one may disagree with his emphasis on topics such as species concepts, species reality, and mechanisms for the evolution of species. However, given that several of today's debates are still about almost identical issues, it seems quite unnecessary to argue that Darwin was muddled, or that he didn't really write a book about speciation at all, as Mayr, and now Coyne and Orr have claimed. It may have been expedient to argue that Darwin hadn't 'got' speciation in the 1940s, but Darwin's work is no longer a very strong competitor for a book published in 2004. If anything, as a number of recent authors have argued, we are today returning to a more Darwinian viewpoint than at any time in the last 60 years, and in this I am including Coyne and Orr's own synthesis. In 'Speciation', we actually see very little treatment of a number of the more antiDarwinian ideas of the 1940s. For instance, Mayr's views that species are necessary for evolutionary diversification, that species are vehicles for the 'storage and protection of variation', that species are group-selected hyper-individuals that exist as 'harmoniously interacting gene complexes', and exhibit 'unity of the genotype', etc, are all dropped. So can we please now reinstate Darwin to his rightful place as the originator of speciation theory, and author of major advances in this area?

\section{(2) The reality of species}

Darwin certainly wasn't arguing that species were 'unreal' in the sense that you couldn't see close parallels between local folk taxonomies and a natural classification. 'To sum up, I believe that species come to be tolerably well-defined objects, and do not at any one period present an inextricable chaos of varying and intermediate links' (Darwin, 1859, p 177). Darwin was, after all, a taxonomist, and had to face practicalities of species delimitation as well as to develop an evolutionary species concept. As I explain above, he was really arguing against creationists for what is today a relatively uncontroversial potential of geographic races and other 'varieties' to evolve into species; as a naturalist, he didn't try to argue that species didn't exist or were completely continuous in sympatry. In fact, his theory of speciation hinged on the extinction of intermediates between species; this was how, to Darwin, species came to be 'tolerably well-defined' morphological clusters.

Yet, Coyne and Orr attempt in a fairly large section of their book to show that Darwin, as well as those who today doubt the distinctness or 'reality' of species, are wrong (pp 10-25, 48-54). Coyne and Orr's main argument for species 'reality' is that folk taxonomies identify the same biological units as modern taxonomies. However, this was never really at issue (and it certainly doesn't prove that the biological species concept is best see below). Instead, it is genuinely hard to decide which actual taxa occurring over large regions of time and space are species, and which are not, as Darwin, and indeed any traveller with a set of 21st century bird, plant, or butterfly guides from different countries will well know. Another problem is that the precise level of natural hybridization required to sink species is always going to be somewhat subjective. Indeed, Coyne and Orr (pp 39-45) clearly recognize these 'unrealities' of actual species, as indeed their predecessors Mayr and Darwin had done. For example, Mayr writes on geographic isolates (1982, p 282): 'The decision whether to call such [allopatric] populations species is somewhat arbitrary'.

One of the thornier issues is that species used to be regarded in the $1940 \mathrm{~s}$ as real entities maintained by special species-level traits, 'isolating mechanisms' (see also 3, below). Coyne and Orr, however, now prefer the less loaded term 'isolating barriers'. They agree that reproductive isolation does not consist of a special set of species-level traits. Instead, 'we maintain that disruptive selection and reproductive isolation are two sides to the same coin' ( $p$ 31). This is a clear rapprochement towards a more Darwinian view that species might, at least sometimes, arise and be maintained by natural selection of a kind similar to that within species.

In the end, I think the debate about species reality boils down, sadly, to different interpretations of the word 'real'. When some of us write that species are 'unreal', or not essentially different from geographic races, the Mayr-Dobzhansky-influenced school feel bound to disagree, even though in today's battles with creationists, it would be a lot simpler to adopt a Darwinian 'species are not special' stance wholeheartedly. To Coyne and Orr, species are 'real' and special because they can mostly be identified as morphological and genetic clusters locally (even though they cannot easily be discriminated across wide geographic areas), and to them the pro-'unreal' Darwinian school appear to argue that species do not exist in this sense at all. Darwinist 'unrealists' are perhaps seen as wreckers, anarchic nihilists intent on destroying hard-won evolutionary consensus. Instead, the differences between the two camps truly depend much more on subtle shades of meaning than perhaps either side, in their caricatures of opponents' positions, would like to admit.

\section{(3) Species as reproductively isolated units}

It will come as no surprise to Coyne and Orr aficionados that they continue to support the view that the biological species concept, in which species are reproductively isolated, is the best. Much of their 45-page chapter 1 is devoted to the topic, and they amplify this viewpoint into a full-length review of several major alternative species concepts in a 35-page appendix. Coyne and Orr here detail the reasons why they adopt reproductive isolation as the key to species, and why they reject genealogical and phylogenetic species, and the simpler view that species are merely clusters of genotypes. The 
biological species concept answers (p 26) 'the most important species problem', which is 'why do sympatric, sexually reproducing species fall into discrete clusters?' Defining species via reproductive isolation dictates a programme of research, where 'reproductive isolation is the proper focus for the study of speciation' (p 32).

Coyne and Orr also recognize that hybridization and introgression occur between biological species much more commonly than previously thought (pp 41-45). 'In our view, distinct species are characterized by substantial but not necessarily complete reproductive isolation' ( $p$ 30). Of course, some organisms largely have uniparental or clonal reproduction, such as many bacteria. Can they form species, even though they lack the means to have reproductive isolation, since they do not mate? Coyne and Orr argue that in bacteria and similar organisms, clusters of genotypes may be maintained by ecological adaptation or other cohesion forces, rather than by reproductive isolation. 'If this is the case, both the cohesion and ecological concepts seem appropriate for dealing with bacterial taxa' ( $\mathrm{p} 45)$.

It seems clear from all this that Coyne and Orr agree that species are in fact clusters of genotypes, but that they prefer to include within their species 'concept' an indication of the processes that maintain this clustering, since it is these factors that must be studied in order to understand how clusters arise. Because they are more interested in the evolution of reproductively isolated, sexual species than in other kinds of speciation, involving bacteria, say, they view reproductive isolation as the important thing about speciation. Essentially, this is exactly what their opponents, who argue that species are more properly treated as clusters of genotypes, are saying as well, only their philosophical preference is to separate the processes of species maintenance from the criterion to identify the groups of individuals on which these processes act. Taxonomic applications of biological species concept and genotypic clusters will be very similar, although Coyne and Orr argue that some distinguishable clusters of genotypes, such as host races of phytophagous insects, which hybridize rather too frequently for their liking, are not separate species. The chief difference is Coyne and Orr's philosophical preference for a 'species concept', which has a mode of species maintenance inherent in its definition of species, rather than a purely taxonomic criterion for species. Logically and practically, however, the two camps are now singing from the same hymn sheet. Perhaps we should all consider this debate is solved, and get on with studying speciation!

\section{(4) Allopatric speciation as the null hypothesis}

Coyne and Orr argue ( $p$ 84) 'that allopatric speciation should be considered the 'default' mode of speciation because it is supported by substantial evidence and occurs under a wider array of conditions than do other modes'. In their view, therefore, allopatry is the most likely hypothesis in any case where allopatric speciation has not been definitively rejected. This view that allopatric speciation should be a null hypothesis, to be tacitly accepted when no other hypothesis was disproved, was previously implicit in the works of Ernst Mayr, and was explicitly enunciated by Futuyma and Mayer (1980). In this book, there has been excellent overall coverage of both theory and new data for sympatric and parapatric speciation, so I was somewhat disappointed not to see a more even-handed approach to conclusions from such studies.

There are several threads to this argument that seem worth investigating: (1) the meaning of allopatry vs parapatry and sympatry; (2) the meaning of allopatric speciation; (3) whether 'accepting' a null hypothesis is ever a good idea in scientific inference; and (4) whether allopatric speciation is indeed more likely. It is my belief that when these difficulties are teased apart, the argument for the primacy of allopatric speciation becomes much less clear.

First, Coyne and Orr follow Gavrilets (2003) in defining allopatry as complete separation, so that in population genetic terms, allopatric speciation occurs when $m$ [ie the fraction of genes exchanged] is effectively zero from the beginning' (Coyne and Orr, p 86). Similarly, two populations are sympatric if they exchange genes at random, that is, are in the same populations, so that the fraction of genes exchanged will be $m=0.5$. For example, in 'Speciation' ( $p$ 203), speciation of host races is said to be sympatric only if different host trees utilized by a diverging pair of populations have completely overlapping times of flowering. All other intermediate levels of gene flow (ie $0<m<0.5$ ) are then defined as parapatry; for this reason, parapatric speciation can be considered the most general form of speciation (Gavrilets, 2003). This brings up a theoretical problem, which is that since speciation is complete only when $m$ is effectively zero, according to Coyne and Orr's biological species concept, only the first genetic change that causes reproductive isolation in diverging populations can be truly said to occur in sympatry, while all remaining genetic changes must be deemed to have evolved in parapatry. Even worse, in this book, parapatry is often treated as 'effective' allopatry, for example in the discussions of comparative evidence for speciation from range distributions of pairs of sister species (see below). Therefore, almost the complete process of sympatric speciation of two populations in contact might be considered allopatric (or perhaps 'microallopatric'). It seems to me that a more interesting question is whether adaptive radiation and speciation is likely and can be driven by natural selection alone, or whether it usually requires the additional deus ex machina of geographic isolation. When put in these terms, rather than in terms of allopatry or sympatry, the question is surely more interesting.

Another, less controversial distinction is between two forms of allopatric speciation, vicariance speciation (the dumbell model, where ranges are split by a major barrier such as a mountain range or ocean) vs peripatric speciation (where a small group of long-distance colonizers encounter new conditions that lead to speciation). 'The only difference between vicariant and peripatric speciation is that in the latter case one population is very small' ( $p$ 105). However, Coyne and Orr are at pains to argue that there is little evidence for founder effect speciation, where genetic drift in small colonizer populations is involved (see pp 387-410). 'Founder events are uncontroversial, but founder effects are not' $^{\prime}(\mathrm{p}$ 106). Instead, in their view, colonization and founder events are among the strongest evidence for allopatric speciation (eg pp 84, 106), mainly via natural selection in the new environments. 
Second, I still find myself somewhat confused by what Coyne and Orr mean by 'allopatric speciation', even accepting their view of allopatry. The problem here is that speciation is usually gradual and requires many genetic changes (this is well demonstrated throughout the book), except perhaps for cases of speciation via polyploidy. If part of the evolution of reproductive isolation occurs in allopatry, and another part in sympatry, what is the geographic mode of speciation? An obvious answer would be that there is a mixture of geographic modes, but with their allopatric bias Coyne and Orr regard these as specialized forms of allopatric speciation, in which only a few minor details are perhaps tidied up in sympatry or parapatry; they seem happy with any mixture of geographic modes, so long as it has the prefix 'allo-' in the term somewhere. We therefore have 'allo-parapatric speciation' (sometimes known as speciation by reinforcement, p 112), 'para-allopatric speciation' ( $p$ 112), and even 'allo-sympatric speciation' ( $p$ 162). In general, they are sceptical of any nonallopatric speciation, and tend to regard the important phase of speciation to have occurred in allopatry. For example: 'Rhagoletis pomonella ... was considered a paradigm of sympatric host race formation until recent work showed that critical evolutionary changes might have occurred in allopatry' ( $p$ 176). Here they refer to work showing that chromosomal polymorphisms in both host races are characterized by North American and Mexican rearrangements (Feder et al, 2003). However, there is very good historical evidence that the applefeeding race of $R$. pomonella evolved from the hawthorn race in sympatry, or at least in interdigitated local parapatry, beginning to spread across northeastern USA about 150 years ago. Do we really know what the 'critical' evolutionary change was, and given that changes may be driven by sufficiently strong selection with $m>0$, why should we make the assumption that any changes in allopatry were critical at all? It seems to me that theory and empirical evidence now argues for a much more pluralist view of the geographic mode of speciation, and indeed one which might readily occur without pure allopatry at all.

Third, should the null hypothesis of allopatric speciation be considered the default mode to be accepted in the absence of any alternative? In statistical inference, we would not accept the null hypothesis in the absence of its disproof, as most biology students taking a first-year statistics course should know. Rather, in view of the plausibility of sympatric and parapatric speciation (see below), it seems to me that we should be trying to test between the various modes of speciation, rather than starting with a bias or a 'default' hypothesis.

Fourth, and finally, is it really true that (p 85) 'the biological conditions allowing parapatric and allopatric speciation are more restrictive than those allowing allopatric speciation?' Coyne and Orr argue (pp 387410) that speciation is rarely completed by genetic drift or founder effects; instead, natural selection is paramount in their view of speciation, which I regard as a refreshing return to Darwin's own view. But if speciation is mostly driven by selection, then all that is required is that the selection coefficient $(s)$ is larger than the rate of gene flow $(m)$ for any given genetic change. Coyne and Orr's common-sense view is that you need less selection if $m=0$, so that allopatric speciation is more likely.
However, underlying this idea is the presumption that gene flow is high ( $m$ approximately 0.5 ) in non-allopatric speciation, while, since speciation is gradual, selection is low ( $s$ nearly 0 for any given genetic change); in other words, their view that allopatric speciation is most likely depends on presuppositions about the likely distributions of $m$ and $s$ in natural populations. However, there are very many situations where individuals and most populations undoubtedly have $m>0$. Most populations are in parapatry and sympatry, and this is much more common than pure allopatry. If speciation is driven by such things as competition for resources, enemy-free space, sexual conflict, or sexual selection, we might also expect that selection is often high, say $s>0.01$ for any single genetic change. Under these situations, we might expect sympatric and especially parapatric speciation to be rather more likely than allopatric speciation, because the overall frequency of genetic variation with high selection coefficients is much higher in parapatry and sympatry than in allopatry.

These arguments should apply as much to hybrid sterility and inviability as to pre-mating or ecological adaptations. In Coyne and Orr's view, 'intrinsic postzygotic isolation ... cannot evolve in sympatry because the genes involved would be purged from interbreeding populations... We suggest, then, that sympatric taxa showing both gene flow and intrinsic postzygotic isolation must have experienced an extended period of allopatry or parapatry' ( $p$ 177). However, it has long been known that most cases of postzygotic barriers evolve as a pleiotropic by-product of other changes in the genome, changes that probably are positively selected (pp 267281). As such, they should often be able to evolve in parapatry and also sympatry fairly easily $(m>0)$.

What about empirical evidence? 'Theoretical studies, which generally show that sympatric speciation occurs quite readily, stand in stark contrast to the paucity of evidence of sympatric speciation in nature ${ }^{\prime}$ ( $p$ 177). However, the argument from nature depends on using allopatric speciation as the 'default' mode. Coyne and Orr support the idea that island speciation implicates allopatry. 'As Darwin (1859) first recognized, novel, species-rich groups endemic to isolated islands and archipelagos prove the occurrence of peripatric speciation after colonization' (p 106). Similarly, at least 50\% of the Hawaiian Drosophila, many of which are confined to individual islands, are inferred to have speciated by an island-hopping peripatric mode ( $p$ 108). But is this true? Suppose I take a somewhat perverse view that sympatric or parapatric speciation is the most likely mode of speciation, or that reinforcement in the presence of gene flow is essential to cause rapid speciation. Then, the Hawaiian sister species on different islands would have to have speciated via a different route: colonization, sympatric or parapatric divergence and speciation, followed by extinction of one of the daughter species. Extinctions are common on islands, and given that speciation has been demonstrably rapid in Hawaii, this doesn't seem that improbable to me. At the very least, these examples show that inferences about peripatric speciation depend strongly on accepting the null hypothesis that speciation is allopatric.

It is also true that the spread of adaptations will be inhibited by allopatry, since useful novel adaptations cannot be exchanged. This will undoubtedly cause 
divergence, but speciation, especially adaptive speciation, will not necessarily be faster. It might often be slower than in non-allopatry because the variation required for adaptive speciation could be more limited. Indeed as Darwin himself noted, islands tend to be less diverse overall than mainlands, and more prone to invasion by continental weedy species. In conclusion, this simple evidence for allopatric speciation is not as simple as it might at first appear.

Empirical evidence had been in the doldrums until a major lifeline to allopatric speciation was thrown aboard by recent comparative methods, particularly those employing the method of Barraclough and Vogler (2000) and its derivatives. Briefly, if the most closely related sister species are sympatric, then it appears that speciation could have been in sympatry; if the closest sister species are allopatric, while there is a tendency for increasing sympatry for older species pairs, then allopatric speciation is deemed more likely. In 'Speciation', these methods are heavily used to support the role of allopatry (pp 72-82). Several points can be made about these methods. Firstly, they depend strongly on species concept. The biological species concept grew out of the so-called 'polytpic species concept' of the period 18901910 , in which geographically differentiated forms could be designated as subspecies within an overall biological species, provided they intergraded at their boundaries or, if completely allopatric, were deemed likely to do so in the event of future contact. More recently, various phylogenetic species concepts have argued that geographically divergent populations with fixed differences should be deemed separate species, even if not reproductively isolated. This new view has led to considerable taxonomic inflation in charismatic vertebrates such as mammals and birds, while many lesser known taxa in groups such as ants and flowering plants have been classified in this way all along (Isaac et al, 2004). Thus, one problem for the Barraclough and Vogler method is the conflict between actual taxonomic and 'true' speciation; under the phylogenetic concept, more allopatric speciation would be inferred; under a strict biological concept, many sister species would become sympatric, and sympatric speciation would be more common.

Another problem concerns the definition of allopatric speciation, and what the 'critical' phase of speciation is in these studies (see above). Supposing rapid speciation requires reinforcement, for example, would we not consider reinforcement to be the critical feature of speciation, and one that must occur during a period of range overlap? Divergence into subspecies in the face of gene flow in parapatry is uncontroversial; what must evolve subsequently is an ability to overlap, in order to make a good reproductively isolated species. This might occur much more rapidly in parapatry and narrow sympatry than in pure allopatry. A third problem is what is meant by allopatry (again, see above): in many cases, sister species are parapatric, so that any apparent support for 'allopatry' is in fact better interpreted as 'not perfectly sympatric'.

Overall, empirical evidence from nature confirms the importance of geography for speciation, but it is a considerable leap of faith to accept the stronger view that complete allopatry is necessary or even most likely in speciation.

\section{Conclusion}

This excellent book contains or refers to most of the information ever published on speciation, including theory, experimental results, and evidence from nature. I have discussed several aspects that particularly interest me, but there are major useful sections I cannot cover, on topics such as chromosomal evolution, ecological isolation, sexual selection, Dobzhansky-Muller incompatibilities, and macroevolution. The basic ingredients are extremely well balanced in a very enticing meal, and my criticisms are mostly about the spices and flavouring, or, if you will, the 'spin' placed on the facts. Yet, by making the strongest possible case for a number of somewhat traditional positions, and indeed highlighting predictions based on these positions, the authors have produced a very useful book for those interested and involved in testing theories of speciation.

\section{References}

Barraclough TG, Vogler AP (2000). Detecting the pattern of speciation from species-level phylogenies. Am Nat 155: 419-434.

Darwin C (1859). On the Origin of Species by Means of Natural Selection, or the Preservation of Favoured Races in the Struggle for Life, 1st edn. John Murray: London.

Feder JL, Berlocher SH, Roethele JB, Dambrowski H, Smith JJ, Perry WL et al (2003). Allopatric genetic origins for sympatric speciation in Rhagoletis. Proc Natl Acad Sci USA 100: 1031410319.

Futuyma DJ, Mayer GC (1980). Non-allopatric speciation in animals. Syst Zool 29: 254-271.

Gavrilets S (2003). Models of speciation: what have we learned in 40 years? Evolution 57: 2197-2215.

Isaac NJB, Mallet J, Mace GM (2004). Taxonomic inflation: its influence on macroecology and conservation. Trends Ecol Evol 19: 464-469.

Mayr E (1942). Systematics and Origin of Species. Columbia Univ. Press: New York.

Mayr E (1982). The Growth of Biological Thought. Diversity, Evolution, and Inheritance. Belknap: Cambridge, MA.

J Mallet

Galton Laboratory, University College London, 4 Stephenson Way, London NW1 2HE, UK E-mail: J.MALLET@UCL.AC.UK 